\title{
Contributions to in-plane piezoresponse on axially symmetrical samples
}

\author{
F. Peter, ${ }^{\text {a) }}$ A. Rüdiger, R. Waser, and K. Szot ${ }^{\text {b) }}$ \\ Institut für Festkörperforschung (IFF) and cni-Center of Nanoelectronic Systems for Information \\ Technology, Forschungszentrum Jülich, 52425 Jülich, Germany \\ B. Reichenberg \\ aixACCT Systems GmbH, 52068 Aachen, Germany
}

(Received 12 May 2005; accepted 5 September 2005; published online 11 October 2005)

\begin{abstract}
We report on the influence of system-immanent asymmetries on the interpretation of in-plane piezoresponse force microscopy (PFM). As PFM is a surface scanning method, the electromechanical interaction of probe tip and sample is a key aspect of all experiments. An initial characterization of topography, surface state, and conductivity is mandatory to separate their signal from the response due to an in-plane polarization state. Our findings underline that any reduction of radial symmetry in the tip-sample system creates an otherwise symmetry-prohibited in-plane signal. (C) 2005 American Institute of Physics. [DOI: 10.1063/1.2090367]
\end{abstract}

Lead-based ferroelectrics have a wide field of applications from nonvolatile memories to electromechanical transducers. By means of a deflected laser beam their piezoelectric response can simultaneously be monitored as a vertical and a torsional deformation of an atomic force microscope (AFM) cantilever. ${ }^{1,2}$ These deflections correspond to an outof-plane and an in-plane deformation of the material under investigation, but occur only when the tip is in motion. In other words, any symmetric deformation around the tip that cancels out is impossible to monitor. For example, on a thin film with a polarization perpendicular to the surface, no torsional deformation should be monitored. However, a different situation is given in Fig. 1. Here piezoresponse force microscopy (PFM) measurements on (001) orientated $\mathrm{Pb}\left(\mathrm{Zr}_{0.52}, \mathrm{Ti}_{0.48}\right) \mathrm{O}_{3}$ (PZT) prepared by chemical solution deposition are shown. On top the single-crystalline nanoislands are flat whereas both the in-plane and the out-of-plane piezoresponse are modulated considerably. A possible microscopic origin of these modulations is discussed in Ref. 3 where the edge dislocations of the sample-substrate interface are observed by transmission electron microscopy (TEM). The piezoresponse measurements are performed with a commercial JEOL JSPM 4210 AFM with PtIr-coated cantilevers from Nanosensors; a voltage of $1 \mathrm{~V}$ at $7 \mathrm{kHz}$ is applied to the cantilever.

The following considerations are related to the technologically relevant case of a tetragonal sample with the polarization perpendicular to the substrate. This results in a fourfold symmetry on the surface. Our findings also hold true in a symmetry-reduced system with the polarization vector along other directions that require a more general but less instructive description of the phenomena. ${ }^{4}$

We monitor the piezoelectric signal as amplitude and phase of the elastic response to a modulated electrical excitation $^{5}$ to obtain information about the piezoelectric ten-

\footnotetext{
${ }^{a)}$ Electronic mail: f.peter@fz-juelich.de

b) Also at Institute of Physics, University of Silesia, 40-007 Katowice, Poland.
}

sor elements $d_{i j k}$ and the polarization orientation $\vec{P}$. Ambiguity arises when other than the intrinsic effects contribute to the signal. The direction of the cantilever axis is $x_{1}$, the detectable in-plane deflection is along $x_{2}$, and the vertical direction is denoted $x_{3}$ (see Fig. 2). The tip will also experience an in-plane deflection along $x_{1}$ and thus cause crosstalk to the out-of-plane signal which is about 20 times less amplified than the in-plane signal. ${ }^{6}$

We now discuss several scenarios that induce an in-plane tip deflections even though the piezoelectric tensor remains globally homogeneous.

(1) Surface topography: We recently discussed the contribution of the topography on the in-plane piezoelectric response. ${ }^{7}$ Any slope is an imbalance of material around the tip. While on one side the material responds to the field, the tip is free to move in the opposite direction. This causes a clearly visible perimeter enhancement. Ac-
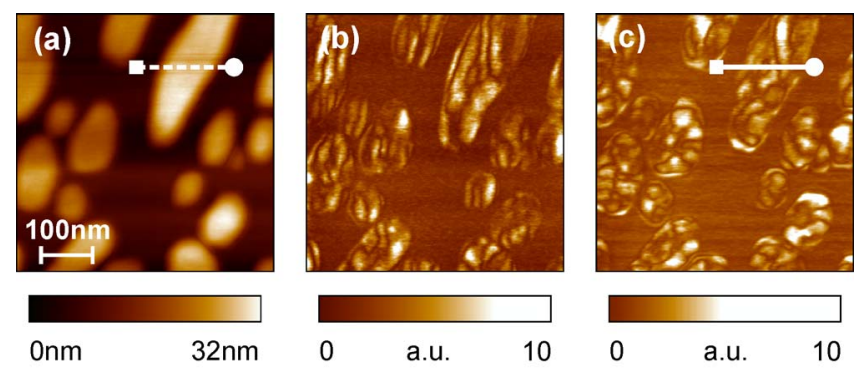

(d)

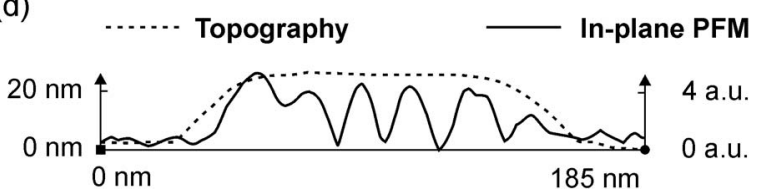

FIG. 1. (Color online) Topography [part (a)], out-of-plane piezoresponse [part (b)], and in-plane piezoresponse [part (c)] of PZT (001) orientated nanograins. Part (d) shows a profile of the topography and in-plane piezoresponse indicated by the lines in parts (a) and (c). Although the (001) orientated grain is flat within experimental errors, the in-plane piezoresponse varies drastically. 


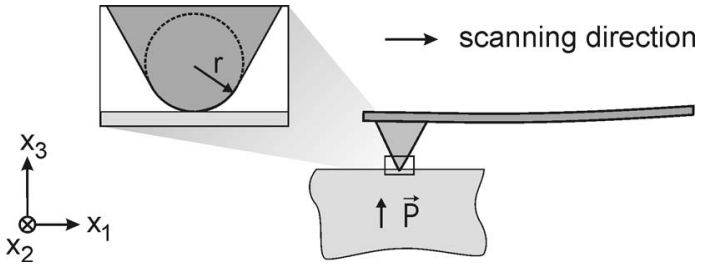

FIG. 2. Illustration of the AFM-tip geometry on a piezoelectric crystal polarized in $x_{3}$ direction.

cording to our finite element simulation the increase of the in-plane field $E_{1}$ and the corresponding coupling to $d_{15}$ for the out-of-plane movement plays a minor role due to the clamping at the interface. Despite a homogeneous piezoelectric tensor, this effect pretends an enhanced piezoelectricity along the perimeter that needs to be carefully separated from strain interaction or etch damage.

(2) Asymmetric tip apex: the maximum electric field strongly depends on the curvature of the PFM probe tip. ${ }^{8}$ For typically utilized tips this radius is about $25 \mathrm{~nm}$ but not necessarily over the full range of the radial angle $\theta$ as depicted in the scanning electron microcopy images of a PtIr and a $\mathrm{W}_{2} \mathrm{C}$ tip in Fig. 3. In a worst-case scenario this also includes the possibility of a complete loss of metal coating of the tip. To assure a reasonably round apex it is worthwhile scanning a reference structure. This structure needs to have sharp features to scan the tip but should be sufficiently soft not to scratch off the platinum coating. Finite element simulations of PFM measurements with an asymmetric tip are given in Fig. 4. As a piezoelectric, a 200-nm-wide, 24-nm-thick $\mathrm{BaTiO}_{3}$ thin film with dielectric constants $\varepsilon_{11}=1500$ and $\varepsilon_{33}=75$ (Ref. 9) has been used. Part (a) shows the potential distribution if a voltage of $-1 \mathrm{~V}$ is applied to the cantilever, part (b) illustrates the in-plane piezoresponse of a symmetrical, and part (c) the in-plane response of an asymmetrical tip. In case of the symmetrical tip the material deformation around the tip cancels out. A high piezoresponse can be seen in the case of a tip with an
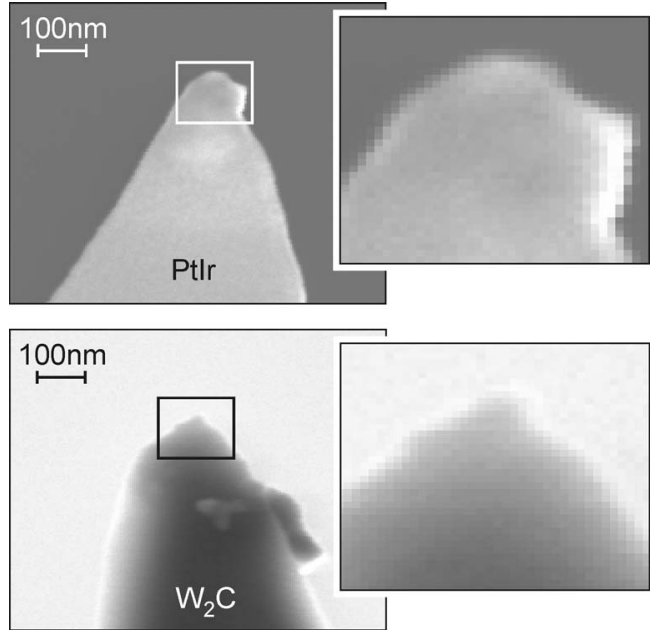

FIG. 3. Scanning electron microscopy images of a used PtIr-coated (top images) and a new $\mathrm{W}_{2} \mathrm{C}$-coated (bottom images) AFM tip.

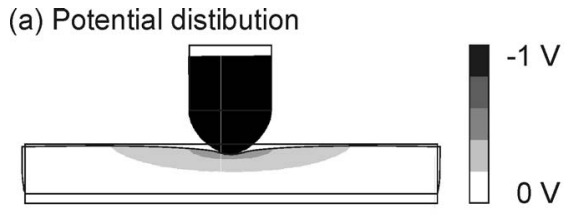

(b) In-plane Piezorespone Symmetrical tip

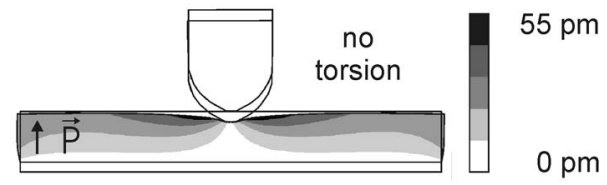

(c) In-plane Piezorespone Asymmetrical tip

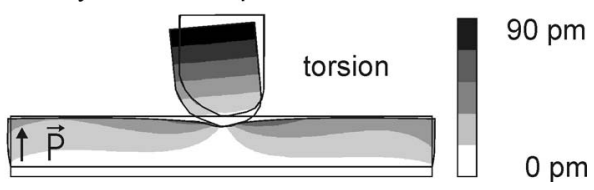

FIG. 4. Simulation of the influence of different tip radii on the in-plane piezoresponse of a symmetric $\mathrm{BaTiO}_{3}$ single crystal. Part (a) shows the potential distribution, part (b) the in-plane piezoresponse in case of a symmetrical tip, and part (c) the in-plane piezoresponse in case of an asymmetrical tip. The piezoelectric deformation has been exaggerated to guide the eye.

approximated radius of $16 \mathrm{~nm}$ on the right and $24 \mathrm{~nm}$ on the left.

(3) Locally varying material parameters: All samples, including single crystals, are imperfect to a certain degree. Local variations in stoichiometry, mechanical properties, or the dielectric constant cannot be ruled out completely. As an illustration, we present a change in the dielectric constant. A section of the setup for the finite element simulation is shown in Fig. 5. We simulate a 400-nm-

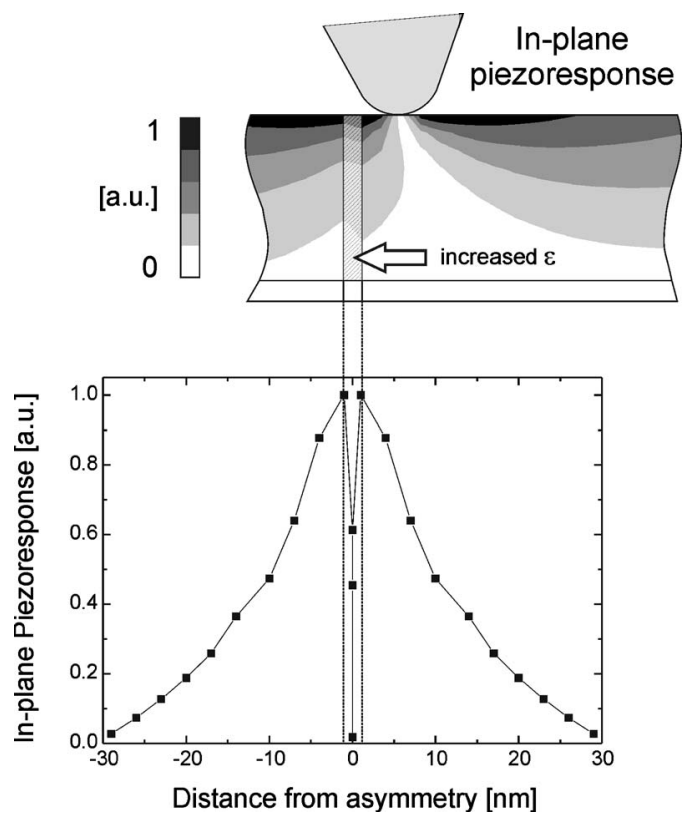

FIG. 5. Simulation of the influence of an increased dielectric constant along $x_{3}$ on the in-plane piezoresponse. The top part shows the piezoresponse amplitude distribution at the indicated tip position. In the bottom part the detectable in-plane response depending on the tip position is depicted. 
(a) Potential distribution

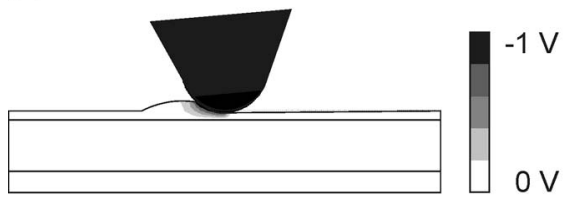

(b) In-plane piezoresponse

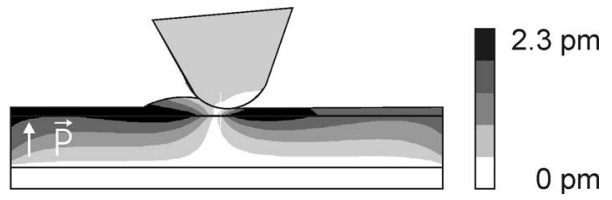

FIG. 6. Simulated potential distribution (a) and in-plane piezoresponse (b) of a $\mathrm{BaTiO}_{3}$ single crystal in the presence of a bow wave.

wide and 30-nm-thick $\mathrm{BaTiO}_{3}$ layer contacted by a large electrode on the bottom and a point electrode on top. In the middle of the crystal a 3-nm-wide plane has been introduced with a $\varepsilon 10 \%$ above the bulk value also resulting in a changed polarization. A voltage of $-1 \mathrm{~V}$ is applied to the point simulating the cantilever. In the top part of the figure the in-plane piezoresponse is given for the case where the cantilever is at the indicated position. The bottom part of the figure shows the in-plane piezoresponse at the point of contact as a function of the tip position. Depending on the local dielectric permittivity, mechanical constants and the tip radius, either rings or circles of in-plane response are created around these spots. Once the needle is in the center of the varied area the symmetrical situation requires the signal to disappear.

Our simulations (Fig. 5) indicate that the range in which the in-plane movement varies is less than $1 \mathrm{pm}$ in the presented case. As this situation has not yet been experimentally observed, we assume our experimental resolution is insufficient to resolve it.

Two other scenarios also reduce the symmetry of the tip and cause a deflection of the tip but only along $x_{1}$ where the in-plane signal only adds to the out-of-plane signal. To what extent this extra contribution is significant is under investigation.

(1) Bow wave of surface adsorbates at the tip apex: Surface adsorbates have an immediate influence on quantitative piezoelectric measurements as they considerably reduce the applied field between tip and sample. ${ }^{10}$ As the tip now propagates parallel to the surface it will create a bow wave (see Fig. 6) in a viscous adsorbate medium. Effectively, this wave enhances the electrical screening in scanning direction and slightly reduces it on the op- posite side. However, this only creates an asymmetry along $x_{1}$ where the in-plane response cannot be detected. A finite element simulation of this scenario is given in Fig. 6. Part (a) shows the potential distribution in the 12-nm-thick $\mathrm{BaTiO}_{3}$ film covered by a $2 \mathrm{~nm}$ adsorbate layer with an dielectric constant of $\varepsilon=8$. The rather small in-plane piezoresponse is presented in part (b).

(2) Space-charge fields: In case a biased needle is moved over a homogeneous semiconductor, the charge carriers will respond with a characteristic time. As the high local fields underneath the tip are beyond the small field approximation for Maxwell relaxation we omit a quantitative analysis. The resulting space-charge field asymetrically screens the field from the tip in the direction of motion $x_{1}$. As previously pointed out the in-plane sensitivity is along $x_{2}$ where this effect does not contribute. These two scenarios imply a speed dependent asymmetry. In case of the space charges the relaxation time has to be compared to the time the tip remains within the area that directly responds to the field. As for the bow wave nothing is known about the viscosity of these surface adsorbates and their adhesion to the tip.

This list of possible contributions of the experimental setup to the delicate interpretation of piezoelectric data is not comprehensive but should increase the awareness for the interaction of probe tip and sample.

In conclusion, we have shown that the in-plane piezoresponse on a symmetrical flat surface can exist provided that the symmetry is broken otherwise. This can happen by surface structures on the sample, a broken symmetry of the scanning apex or locally varying material parameters.

The authors would like to thank I. Szafraniak and M. Alexe (within Volkswagen Foundation Grant No. VW I/80897) for providing the PZT sample.

${ }^{1}$ L. M. Eng, H.- J. Güntherodt, G. Rosenman, A. Skliar, M. Oron, M. Katz, and D. Eger, J. Appl. Phys. 83, 5973 (1998).

${ }^{2}$ A. Roelofs, U. Böttger, R. Waser, F. Schlaphof, S. Trogisch, and L. M. Eng, Appl. Phys. Lett. 77, 3444 (2000).

${ }^{3}$ M. Chu, I. Szafraniak, R. Scholz, C. Harnagea, D. Hesse, M. Alexe, and U. Gösele, Nat. Mater. 3, 87 (2005).

${ }^{4}$ C. Harnagea, A. Pignolet, M. Alexe, and D. Hesse, Integr. Ferroelectr. 44, 113 (2002).

${ }^{5}$ S. V. Kalinin, E. Karapetian, and M. Kachanov, Phys. Rev. B 70, 184101 (2004).

${ }^{6}$ F. Peter, A. Rüdiger, R. Waser, K. Szot, and B. Reichenberg, Rev. Sci. Instrum. 76, 046101 (2005).

${ }^{7}$ F. Peter, A. Rüdiger, R. Dittmann, R. Waser, K. Szot, B. Reichenberg, and K. Prume, Appl. Phys. Lett. 87, 082901 (2005).

${ }^{8}$ J. Fleig and J. Maier, Solid State Ionics 86-88, 1351 (1996).

${ }^{9}$ Landolt-Börnstein Condensed Matter III, edited by K. H. Hellwege (Springer, Heidelberg, 1981), Vol. 16A.

${ }^{10}$ F. Peter, K. Szot, R. Waser, B. Reichenberg, S. Tiedke, and J. Szade, Appl. Phys. Lett. 85, 2896 (2004). 